A Standard for the Transmission of IP Datagrams over Ethernet Networks

Status of this Memo

This RFC specifies a standard method of encapsulating Internet Protocol (IP) [1] datagrams on an Ethernet [2]. This RFC specifies a standard protocol for the ARPA-Internet community.

Introduction

This memo applies to the Ethernet (10-megabit/second, 48-bit addresses). The procedure for transmission of IP datagrams on the Experimental Ethernet (3-megabit/second, 8-bit addresses) is described in [3].

Frame Format

IP datagrams are transmitted in standard Ethernet frames. The type field of the Ethernet frame must contain the value hexadecimal 0800 . The data field contains the IP header followed immediately by the IP data.

The minimum length of the data field of a packet sent over an Ethernet is 46 octets. If necessary, the data field should be padded (with octets of zero) to meet the Ethernet minimum frame size. This padding is not part of the IP packet and is not included in the total length field of the IP header.

The minimum length of the data field of a packet sent over an Ethernet is 1500 octets, thus the maximum length of an IP datagram sent over an Ethernet is 1500 octets. Implementations are encouraged to support full-length packets. Gateway implementations MUST be prepared to accept full-length packets and fragment them if necessary. If a system cannot receive full-length packets, it should take steps to discourage others from sending them, such as using the TCP Maximum Segment Size option [4] .

Note: Datagrams on the Ethernet may be longer than the general Internet default maximum packet size of 576 octets. Hosts connected to an Ethernet should keep this in mind when sending datagrams to hosts not on the same Ethernet. It may be appropriate to send smaller datagrams to avoid unnecessary fragmentation at intermediate gateways. Please see [4] for further information on this point. 


\section{Address Mappings}

The mapping of 32-bit Internet addresses to 48-bit Ethernet addresses can be done several ways. A static table could be used, or a dynamic discovery procedure could be used.

\section{Static Table}

Each host could be provided with a table of all other hosts on the local network with both their Ethernet and Internet addresses.

Dynamic Discovery

Mappings between 32-bit Internet addresses and 48-bit Ethernet addresses could be accomplished through the Address Resolution Protocol (ARP) [5]. Internet addresses are assigned arbitrarily on some Internet network. Each host's implementation must know its own Internet address and respond to Ethernet Address Resolution packets appropriately. It should also use ARP to translate Internet addresses to Ethernet addresses when needed.

\section{Broadcast Address}

The broadcast Internet address (the address on that network with a host part of all binary ones) should be mapped to the broadcast Ethernet address (of all binary ones, FF-FF-FF-FF-FF-FF hex).

The use of the ARP dynamic discovery procedure is strongly recommended.

Trailer Formats

Some versions of Unix 4.2bsd use a different encapsulation method in order to get better network performance with the VAX virtual memory architecture. Consenting systems on the same Ethernet may use this format between themselves.

No host is required to implement it, and no datagrams in this format should be sent to any host unless the sender has positive knowledge that the recipient will be able to interpret them. Details of the trailer encapsulation may be found in [6].

(Note: At the present time Unix 4.2bsd will either always use trailers or never use them (per interface), depending on a boot-time option. This is expected to be changed in the future. Unix 4.2bsd also uses a non-standard Internet broadcast address with a host part of all zeroes, this may also be changed in the future.) 
Byte Order

As described in Appendix B of the Internet Protocol

specification [1], the IP datagram is transmitted over the Ethernet as a series of 8 -bit bytes.

References

[1] Postel, J., "Internet Protocol", RFC-791, USC/Information Sciences Institute, September 1981.

[2] "The Ethernet - A Local Area Network", Version 1.0, Digital Equipment Corporation, Intel Corporation, Xerox Corporation, September 1980 .

[3] Postel, J., "A Standard for the Transmission of IP Datagrams over Experimental Ethernet Networks", RFC-895, USC/Information Sciences Institute, April 1984.

[4] Postel, J., "The TCP Maximum Segment Size Option and Related Topics", RFC-879, USC/Information Sciences Institute, November 1983.

[5] Plummer, D., "An Ethernet Address Resolution Protocol", RFC-826, Symbolics Cambridge Research Center, November 1982.

[6] Leffler, S., and M. Karels, "Trailer Encapsulations", RFC-893, University of California at Berkeley, April 1984. 